\title{
Lipid Analysis of Developing C. sativa Seeds and Cultured Embryos
}

Mike Pollard*, Tina M. Martin and Yair Shachar-Hill

Department of Plant Biology, Michigan State University, 612 Wilson Rd., East Lansing, Michigan 48824

Submit to Phytochemistry

Section: Metabolism

Corresponding author: pollard9@msu.edu

Dedicated to Professor Frank D. Gunstone, who was Mike Pollard's PhD advisor.

\begin{abstract}
C. sativa is a cultivated oilseed rich in triacylglycerols containing oleic, linoleic, $\alpha$-linolenic and eicosenoic acids. As it holds promise as a model species, its lipid synthesis was characterized in vivo and in culture. Lipid accumulates at a maximum rate of about $26 \mu \mathrm{g} / \mathrm{day} / \mathrm{seed}(11.5 \mathrm{mg}$ lipid/day/gram fresh seed weight), a rate comparable with other oilseeds. Noteworthy is a late stage surge in $\alpha$-linolenic acid accumulation. Small amounts of unusual epoxy and hydroxy fatty acids are also present in the triacylglycerols. These include 15,16-epoxy- and 15-hydroxyoctadecadienoic acids and homologous series of $\omega 7$-hydroxy-alk- $\omega 9$-enoic and $\omega 9 / 10$-hydroxyalkanoic acids. Mid-maturation embryos cultured in vitro have growth and lipid deposition rates and fatty acid compositions that closely match that of seeds, but extended culture periods allow these rates to rise and surpass those observed in planta. Optimized thin layer chromatography systems for characterization of labeled products from acetate or glycerol labeling are described. Glycerol label is only found in acylglycerols, largely as the intact glyceryl backbone, but acetate can label acyl groups and sterols, the latter to a much higher relative specific activity. This presumably occurs because mevalonic acid precursor is derived from the non-plastid pool of acetyl-CoA that is also the source for malonyl-CoA to drive FAE1-dependent chain elongation. Particular attention has been paid to the separation of sterols and diacylglycerols, and to hydrogenation of triacylglycerols to simplify their analysis. These improved methods will allow more accurate analyses of the fluxes of lipid metabolism in cultured plant embryos.
\end{abstract}

Keywords: Camelina sativa; Brassicaceae; seed development; embryo culture; rates of lipid accumulation; linolenic acid 


\section{1: Introduction}

Camelina sativa has a long history as an Old World seed crop. It has recently been introduced into the US where it is regarded as a new industrial oilseed crop and is grown largely in the Pacific Northwest for the potential purpose of producing oil to be converted into biofuel (methyl and/or ethyl fatty acid esters) [Frohlich and Rice, 2005; Moser and Vaughn, 2010]. It fits the need for lower inputs and intercropping in dry land farming. C. sativa has also become a useful model to study oil biosynthesis [Horn PJ, 2013; Kang et al., 2011; Liang et al., 2013; Mansour et al., 2014; Mudalkar et al., 2014]. A member of the Brassicaceae, it has several advantages over Arabidopsis thaliana. Like Arabidopsis, genetic transformation is facile [Lu and Kang, 2008], and several generations can be grown in a year. However, it produces seeds that are about 50 times larger than those of Arabidopsis, making it preferable for biochemical studies. Thus Camelina is also attractive for oilseed engineering experiments because promising lines can be readily scaled up to produce significant quantities of test oils [Liu et al, 2015]. Given these advantages, it is timely to develop a quantitative flux map for lipid biosynthesis in developing Camelina embryos.

The pathways of fatty acid and triacylglycerol (TAG) synthesis in developing seeds have been extensively investigated, with biochemical and genetic studies providing information for genetic engineering of oilseed crops to produce more oil, to modulate fatty acid composition, or to produce novel oils, usually containing uncommon fatty acids. Enzymes responsible for unique facets of fatty acid metabolism, including downstream acyl transferases, have been identified and the corresponding genes cloned and expressed, with various levels of success in optimizing yields of novel oil products [Cahoon et al., 2007; Clemente and Cahoon, 2010; Napier et al., 2014]. However, understanding the lipid metabolism of seeds requires not just a pathway and an accounting of genes, from transcription factors to enzymes, but an understanding of the fluxes and their regulation, particularly if maximizing a particular composition is desired. Unlike central metabolism, where isotopomer imprinting of reporter molecules and mass balances are used to determine fluxes, lipid flux measurements require kinetic labeling. Previously such a study was carried out on cultured embryos from developing soybeans using short term $(\leq 30$ minutes) labeling with ${ }^{14} \mathrm{C}$-substrates, to quantify the substantial cyclic fluxes of acyl groups through membrane lipids [Bates et al., 2009].

To extend such studies to stable isotope labeling with its associated isotopomer analysis of acylglycerols, one pre-requisite is longer duration labeling times. Longer assay times will also provide a very complementary kinetic data set for modeling when added to that from initial rate studies. In this paper, lipid deposition by cultured zygotic embryos from developing $C$. sativa seeds is compared with seed development in siliques on the plant. The cultured embryos closely mirror lipid deposition in planta, both in terms of their rates of lipid accumulation and their fatty acid composition. In addition, minor epoxy- and hydroxy-fatty acids were identified. The presence of these acids is accentuated 3-fold by their distinctive TAG molecular species. Separation methods have been customized to allow better analyses of labeled fatty acids and lipids specifically from Camelina embryos, with particular attention paid to the TLC separation of sterols and diacylglycerols, and to hydrogenation of triacylglycerols to simplify their analysis. In a companion paper [Pollard et al., co-submitted], aspects of long term acetate and glycerol labeling at concentrations sufficient to permit stable isotope isotopomer analysis of acylglycerol products are described. These two papers provide the baseline information required to use cultured embryos for the quantitative analysis of lipid biosynthesis in developing C. sativa seeds. 


\section{2: Results and discussion}

\section{2:1 Fatty acid and lipid accumulation in C. sativa seeds during development}

The embryo is the main storage tissue of $C$. sativa seeds. Knowledge of embryo development, and that of the entire seed, is a foundation for understanding the biochemistry and genetics of lipid deposition. This data was used to define the best age to harvest embryos for culture. Since dissection of embryos from seeds is difficult beyond mid-maturation, seed development was tracked from 5 to 50 days post anthesis (DPA) (Figure 1). At 5 DPA, embryos are at "torpedo" stage or entering "linear cotyledon" stage. They pass through the "green cotyledon" stage at mid-maturation (14-20 DPA), a period where the rate of oil synthesis is maximal, after which the embryos slowly turn yellow and desiccate. Chlorophyll levels peak at 16 DPA, at about $1 \mathrm{mg} / \mathrm{g}$ fresh wt. in the embryos. This peak chlorophyll concentration on a fresh weight basis is about half of that found in $C$. sativa leaves, and indicates a substantial development of photosynthetic machinery in the embryo at mid-maturation. The mid-point for oil deposition occurs at 17-18 DPA. At mid-maturation, the oil accumulation rate is $26 \mu \mathrm{glipid} / \mathrm{seed} /$ day (that is, about 11.5 $\mathrm{mg} \mathrm{lipid/g}$ fresh wt/day). Most of the oil deposition is associated with the embryo and the product is TAG. For comparison, a number of oilseed species grown in the greenhouse had oil deposition rates from 2.75 (soybean) to $40 \mathrm{mg}$ lipid/g fresh wt/day (Cuphea lutea), with safflower and rapeseed at 15.5 and $12.5 \mathrm{mg} \mathrm{lipid} / \mathrm{g}$ fresh wt/day respectively [Singh et al., 1986]. Data presented on Arabidopsis seed development [Baud et al., 2002] allows the Arabidopsis lipid accumulation rate at mid-maturation to be estimated at $19 \mathrm{mg} \mathrm{lipid} / \mathrm{g}$ fresh $\mathrm{wt} /$ day. At maturity, the oil content (dry weight basis) of $C$. sativa seeds from growth chamber plants was $42.1 \pm 1.6$ $\%(n=12)$, which compares with reported values of 29.9 to $38.3 \%$ for 13 accessions of field grown plants [Budin et al., 1995] and 15.5 to $41.7 \%$ for 40 accessions of field grown plants [Rodriguez-Rodriguez et al., 2013]. In the latter study, seed development was also tracked for a line grown in the growth-chamber. The data are broadly similar to those reported here, but the maximum rate was lower $(15 \mu \mathrm{g} \mathrm{lipid/seed/day)} \mathrm{while} \mathrm{the} \mathrm{midpoint} \mathrm{for} \mathrm{oil} \mathrm{deposition} \mathrm{occurred}$ three days later (21 DPA). These differences are likely to be attributed to different cultivars and growth conditions between the studies.

The seed oil of mature, wild type Camelina seeds is rich in polyunsaturated fatty acids, with $\alpha$ linolenic (C18:3) acid being the most abundant fatty acid [Budin et al., 1995; Zubr and Matthus, 2002; Abramovic and Abram, 2005; Rodriguez-Rodriguez et al., 2013]. In common with other members of the Brassicaceae, $C$. sativa seeds contain significant amounts of C20-C24 very long chain fatty acids (VLCFA), primarily eicos-11-cis-enoic acid (C20:1). The accumulations of the individual fatty acids are shown in Figure 2A. Eicos-11-cis-enoic acid is first detected at 11 DPA. It and the other VLCFA are presumably synthesized by an elongase homologous to AtFAE1 [Millar and Kunst, 1997]. FAE1 expression can serve as a marker for seed-specific lipid synthesis [Girke et al., 2000; Ruuska et al., 2002], so the appearance of C20:1 provides a useful determinant for the onset of the seed maturation. The deposition of minor VLCFAs, such as eicosanoic acid (C20:0) and erucic acid (C22:1), closely parallels that of C20:1. The rates of accumulation of individual fatty acids are shown in Figure $2 \mathrm{~B}$. At no time are the rates negative for any fatty acid, which would indicate removal of acyl groups from TAG for further desaturation, elongation or turnover. Of particular note is the accelerating accumulation of C18:3, such that by 22 DPA it constitutes about $60 \%$ of the rate of total fatty acid accumulation. Equally noteworthy is the abrupt drop in the deposition rate of $\mathrm{C} 18: 3$ as the maturation phase draws to a close. The rate of oleic acid (C18:1) deposition shows a strong dip during midmaturation consistent with its role as precursor for linoleic acid (C18:2), C18:3 and C20:1 biosynthesis. Also, in late seed maturation, stearic acid (C18:0) accumulation is greatly reduced relative to palmitic acid (C16:0). 
The fatty acid content and composition of the embryo major lipid classes at 16 DPA are shown in Supplement Table 1. This age was chosen as it is the starting age for embryos in the kinetic experiments (see below, and [Pollard et al., co-submitted]). The data can be used to calculate limiting net flux values for acyl groups moving between lipid classes. Total lipids contained 383 $\pm 24(\mathrm{SD}, \mathrm{n}=3)$ nmoles of fatty acids/embryo, while $326 \pm 17,3.4 \pm 0.1$ and $28.0 \pm 1.4$ nmoles/embryo respectively were recovered in TAG, diacylglycerols (DAG) and polar lipids (PL) (i.e. mainly membrane lipids). The sum of TAG $+D A G+P L=357.1$ nmoles/embryo, which is $\sim 93 \%$ recovery from total lipid acyl groups, but when epoxy- and hydroxy-TAGs (see below) are also included this acyl group recovery increases to 96\%. As TAG is the major component of total lipids, it is not surprising that the fatty acid compositions of TAG and total lipids are very similar. The one fatty acid missing from TAG is hexadeca-7-cis, 10-cis, 13-cis-trienoic acid (C16:3). As also noted by Rodriguez-Rodriguez et al. (2013), C. sativa can be added to the list of C16:3 plants [Mongrand et al., 1998]. In Camelina leaf lipids, C16:3 almost exclusively resides in monogalactosyldiacylglycerol (MGDG, at 34 mole \%). This indicates that a plastid pathway for galactosylglycerolipid synthesis is present. The fact that C16:3 is not found in TAG indicates that diacylglycerol moieties or individual fatty acids from galactosylglycerolipids are very minor precursors for TAG synthesis in the embryo.

At 16 DPA, PL constitute 7-8 mole \% of total acyl groups. Of the total PL pool, the contributions of plastid-localized lipids are: MGDG, 16\%; phosphatidylglycerol (PG), 8\%; diglalactosyldiacylglycerol, $10 \%$; and sulfoquinovosyldiacylglycerol, $5 \%$, to give a plastid acyl lipid content of about 39\% of total PL. The fatty acid compositions for each lipid class are similar to those for leaves. The one exception is that PG does not contain any hexadec-3-trans-enoic acid. The significance of this is unclear, although this trans-fatty acid is present in $C$. sativa leaf PG at 22 mole \%. Since the chlorophyll content of the seeds is already peaking at 16 DPA (Figure 1), it is expected that plastid lipid synthesis is already declining. The other major membrane glycerolipids are phosphatidylcholine (PC) at 45\%, phosphatidylethanolamine at $7 \%$ and phosphatidylinositol at $2 \%$. PC is considered the major site for fatty acid desaturation and for the supply of polyunsaturated fatty acids for TAG synthesis in oilseeds [Bates and Browse, 2012; Bates et al., 2013; Calhoun et al., 2007]. At mid-maturation Camelina seed PC has C18:2 to $\mathrm{C} 18: 3$ to $\mathrm{C} 18: 1$ ratio of approximately 1 to 0.65 to 0.6 . However, at 16 DPA, the accumulation rate in seeds of $\mathrm{C} 18: 2$ to $\mathrm{C} 18: 3$ to $\mathrm{C} 18: 1$ is about 1 to 1.35 to 0.25 (Figure 2B). Thus there must be either significant enrichment processes or distinctive precursor pools for C18:3 incorporation into TAG, relative to C18:1 and C18:2 incorporations.

\section{2:2 Minor fatty acids and the corresponding triacylglycerols present in C. sativa seed oil}

In ${ }^{14} \mathrm{C}$ acetate and glycerol labeling experiments, diffuse but persistent labeled bands with a polarity between TAG and DAG were observed when lipids were analyzed by normal phase silica TLC (see later section, Figure 6B). These bands represented up to $4.5 \%$ of the total label. To identify these fractions, oil from mature $C$. sativa seeds was analyzed by TLC. Preparative TLC gave $91.4 \%$ of the recovered lipid mass as normal TAG. Identified minor constituents, in order of increasing polarity, were epoxy-TAG (1.1\%), hydroxy-TAG (1.4\%), 1,3-DAG (0.2\%), 1,2-DAG (0.3\%), sterol (0.6\%) and the PL fraction remaining at the origin (3.85\%). By comparing $R f$ values, TLC analysis using seed oil standards from Euphorbia lagascae and Ricinus communis confirmed identifications for Camelina mono-epoxy-TAGs and monohydroxy-TAGs, respectively. Base-catalyzed transmethylation of the epoxy-TAG fraction allowed identification of 15,16-cis-epoxy-linoleate as a major fatty acid (Figure 3A), based on its GC retention characteristics on polar and non-polar capillary columns, normal and reversed phase TLC behavior, and its EI mass spectrum (Supplement Figure 1). Homologs of 15,16-cis- 
epoxy-linoleate were not observed; nor was 12,13-cis-epoxy-oleate. 15,16-cis-Epoxy-linoleic acid was previously identified as a minor component in Camelina seed oil [Gunstone and Morris, 1959]. Although its structure, other than its absolute stereochemistry, was fully elucidated, it has apparently not been reported in the compositional analyses of Camelina oils since its identification [Budin et al., 1995; Zubr and Matthus, 2002; Abramovic and Abram, 2005; Rodriguez-Rodriguez et al., 2013]. It represents $0.3-0.4 \%$ of total fatty acid methyl esters (FAMEs). One reason for this may be that on DB-23 capillary GC columns, which are commonly used for such analyses, it has a retention time identical to that for methyl tetracos-15enoate.

Transmethylation of the hydroxy-TAG fraction gave a more complex set of FAMEs (Figure 3B) which included three sets of hydroxy fatty acids. One set constituted the expected products from a fatty acyl $\Delta 12$-hydroxylase and consisted of 12-hydroxyoctadecenoate, 12-hydroxyoctadecadienoate, 14-hydroxyeicosenoate, and 16-hydroxydocosenoate. Presumably the first three are methyl ricinoleate, densipolate and lesquerolate, respectively, as they had retention times identical to these standards and the expected mass spectra. 16-Hydroxydocosenoic acid has been reported as a trace component in Heliophila amplexicaulis seed oil [Plattner et al., 1979]. The mass spectrum for methyl 16-hydroxydocosenoate from Camelina is shown in Supplement Figure 2, Panel B3. Given the bifunctional FAD2-like $\Delta 12$ desaturase-hydroxylase gene described in Lesquerella [Broun et al., 1998], a closely related genus to Camelina, the w6hydroxy-unsaturated fatty acid series probably results from a FAD2 enzyme with low levels of hydroxylase activity.

9(10)-Hydroxystearate, 12(11)-hydroxyeicosanoate, 13(14)-hydroxy-docosanoate, and 15hydroxytetracosanoate constituted the second series. The minor isomer is bracketed. Thus they are a mixed $\omega 9 / \omega 10$ series of saturated hydroxy fatty acids. 9(10)-Hydroxystearate has been identified as a minor component in cutin from the leaves of Rosmarinus officinalis [Brieskorn and Kabelitz, 1971]. The mass spectra for the putative 12(11)-hydroxyeicosanoate and 13(14)hydroxydocosanoate methyl esters are shown in Supplement Figure 2, Panels B1 and B2. The biosynthesis and localization of these C18-C24 saturated hydroxyfatty acids in the seed is unclear. Their biosynthesis might be connected with wax or cutin biosynthesis in epidermal cells, but mid-chain hydroxy fatty acids without an additional $\omega$-hydroxy or $\omega$-carboxy functional group are rare in cutin and not observed in suberin [Holloway, 1984].

The third distinctive hydroxy fatty acid "set" had a sole member, putatively identified as 15hydroxylinoleate (mass spectrum in Supplement Figure 2, Panel B4) [Hamberg and Hamberg, 1996]. It is possible that a FAD3-like gene may exhibit, in addition to its dominant $\Delta 15$ desaturation activity, low levels of $\alpha$-linolenate 15,16-epoxidase activity and/or linoleate 15hydroxylase activity. Since it also was possible that an epoxide hydratase might act upon 15,16epoxylinoleate, 12,13-threo-dihydroxyoleate was prepared from vernolate to use as a standard to estimate the chromatographic properties of 15,16-dihydroxylinoleate. However, 15,16dihydroxylinoleate was not detected.

The levels of total oxygenated fatty acids are estimated as $\sim 1 \%$ of total fatty acids, but their incorporation into TAGs amplifies the mole fraction of corresponding TAG molecular species. Thus a modest correction for TAGs with additional oxygen-containing functional groups (band [O]TAG in Figure 6B) is required for any flux analysis. However, the focus on the minor epoxy and hydroxy fatty acids arose largely from concerns about their potential for interference in mass spectrometry analysis of TAGs. The TAG molecular species profile of Camelina seed oil is exceedingly complex. Given 13 fatty acids ( $\omega 9$ and $\omega 7$ positional isomers are combined Supplement Table 1), there are 2197 stereochemically distinct and 455 compositionally distinct 
TAG molecular species possible. ESI-MS analysis of Camelina oil shows at least 44 distinct molecular species, as defined by m/z value, from C50:n to C62:n TAG molecular species profile ( $C$ for total acyl carbon atoms, $n$ for number of double bonds) (Supplement Figure 3A). Within each $\mathrm{m} / \mathrm{z}$ species, there will be a large number compositionally distinct species. Such complexity will make it much harder to accurately deconvolute the individual isotopomers from a ${ }^{13} \mathrm{C}$ labeling experiment. To circumvent this problem, lipids from ${ }^{13} \mathrm{C}$ labeling experiments have been hydrogenated, greatly simplifying TAG analysis.

ESI-MS of hydrogenated Camelina mature seed oil shows a dominant set of hydrogenated TAG $\left(\mathrm{M}+\mathrm{NH}_{4}\right)^{+}$adducts corresponding to $\mathrm{C} 52: 0, \mathrm{C54}: 0, \mathrm{C56:0}$ and C58:0 species, with minor peaks for C50:0, C60:0 and C62:0 species (Figure 4A, Supplement Figure 3A). There is also a series of smaller peaks eluting at $\sim 14$ mass units between the major peaks. They might be mistaken for TAGs containing odd-chain fatty acids, but in fact the levels of odd-chain fatty acids are negligible in Camelina. Instead, they arise both from minor additional adducts formed in the electrospray process and from epoxy- and hydroxy-TAG species. Epoxy and hydroxy groups are resistant to catalytic hydrogenation and add 14 amu and 16 amu respectively to TAG $\mathrm{m} / \mathrm{z}$ values. Their peak intensity may over-represent their concentration, because TAGs containing oxygenated fatty acids are likely to have a higher efficiency of adduct formation in ESI-MS.

TAG, epoxy-TAG and hydroxy-TAG fractions purified by TLC were hydrogenated and analyzed by ESI-MS (Figure 4A, 4B and 4C respectively). The purified TAG still retained minor adduct formation (Figure 4A). The ESI-MS for purified and hydrogenated epoxy-TAG is dominated by a series of peaks with $\mathrm{m} / \mathrm{z}$ values 14 amu higher than for TAGs (Figure 4B). Furthermore, a parallel analysis conducted with the mono-epoxy-TAG fraction in seed oil from Euphorbia lagascae confirmed that the epoxide ring was resistant to reduction under our hydrogenation conditions (Supplement Figure 3B). The most polar TAG fraction, hydroxy-TAG, contains predominantly hydroxy-TAG but also some epoxy-TAG and epoxy-hydroxy-TAG (Figure 4C). Although epoxy- and hydroxy-TAGs will not interfere with ${ }^{13} \mathrm{C}$ isotopomer analyses of hydrogenated TAGs from labeling experiments, epoxy-hydroxy and dihydroxy-TAGs have the potential to bias such measurements. They might also confound TLC separations of DAG and sterol fractions. For example, consider TAG species with $\mathrm{m} / \mathrm{z}=\mathrm{M}$ and $\mathrm{C}(\mathrm{n})$ acyl carbon atoms. The epoxy-hydroxy and dihydroxy-C(n)-TAGs they would produce $(M+30)$ and $(M+32)$ peaks, respectively, and with enhanced sensitivity using ESI-MS analysis. These would create apparent $(M+2)$ and $(M+4)$ isotopomer peaks for the $C(n+2)$ TAG homolog at $(M+28)$. Fortunately, the concentrations of epoxy-hydroxy- and dihydroxy-TAG species are very low. However, it is a worthwhile precaution to routinely purify TAGs away from [O]TAG prior to ${ }^{13} \mathrm{C}$ isotopomer analysis.

\section{2:3 Growth of embryos in culture}

Conditions for Camelina embryo culture were developed from measurements of the composition of the ovule sac fluid in the developing seeds [Carey, L., Cocuron, J.-C. and Shachar-Hill, Y., unpublished results]. Based on the data provided in Figures 1 and 2, several culturing experiments were performed on embryos harvested from 10 to16 DPA. From these tests, a routine culture protocol for mid-maturation embryos was chosen whereby embryos were harvested at 15 DPA and cultured for a full day before assays were initiated. Figure 5A shows the fresh and dry weight and fatty acid accumulation over a culture period of 12 days for 15 DPA embryos. It also shows results from zygotic embryos harvested at 12-16 DPA (dotted lines), in order to assess progression prior to culture.

During the first 8 days of the culture, both growth, as measured by accumulation of dry weight, and lipid accumulation, increased beyond that observed for seeds. The maximum rates of dry 
weight and fatty acid accumulation were 152 and $78 \mu \mathrm{g} /$ day/cultured embryo respectively. These are greater (x 2.75 and x 3.0 respectively) than the values of 55 and $26 \mu \mathrm{g} /$ day/seed observed for whole seeds in siliques (values which are dominated by the embryo metabolism; Figure 5B). Also during the first 8 days in culture, the moisture content of the embryos increased slightly, while the chlorophyll content remained largely unchanged. This contrasts with seeds (and hence their embryos), where by 23 DPA desiccation has begun and a significant reduction in the amount of chlorophyll was observed. After 8 days in culture, a deceleration in growth and lipid accumulation was observed. The rise in moisture content after 12 days in culture is consistent with some embryos forming root initials.

Fatty acids, as a percentage of total dry weight, increased from $38.0 \%$ at 17 DPA (2 days in culture) to a peak of $48.0 \%$ by 25 DPA. The last three time points gave oil contents that were not significantly different from each other, and when averaged gave $47.5 \% \pm 1.7 \%(n=8)$. Higher oil contents for embryos when compared to those for whole seed have been noted for $A$. thaliana and Brassica napus mature seeds [Li et al., 2006]. Figure 5C shows how the individual fatty acids accumulate over time in culture. This should be compared with Figure 2A. After prolonged culture, the fatty acid composition was C18:3 > C18:1 > C18:2 > C20:1. This particular experiment had higher oleate accumulation than seen in other cultures, where $\mathrm{C} 18: 3$ $>$ C18:1 C18:2 > C20:1 (the variance between cultures is shown in Supplement Table 2). By contrast, the mature seed composition was C18:3 > C18:2 C20:1 > C18:1. The cessation of accumulation of C16:0 (and indeed all saturates), C20:1 (and all C20-24 FA) and C18:3 in culture after 23 DPA was abrupt, while C18:1 and C18:2 accumulations continued at reduced rates. This cessation in accumulation of specific fatty acids occurred in the same order as that seen in seeds (Figure 2A). Also, total fatty acid accumulation rapidly declined between 24 and 27 DPA, which is when the halt in lipid accumulation in seeds occurs. The most striking changes noted for fatty acid composition relate to $\mathrm{C} 18: 3$. The accelerated accumulation of $\mathrm{C} 18: 3$ later in seed maturation (Figure 2B) was also observed in embryo culture (Figure 5C). Likewise, the very abrupt cessation of $\mathrm{C} 18: 3$ deposition in seeds also occurred in cultured embryos and preceded cessation of oleate and linoleate accumulation.

\section{2:4 Analyses of acetate and glycerol labeled lipids from cultured embryos}

A large number of substrates have been used to study lipid biosynthesis in plant tissues, including sugars, water, pyruvate and alanine, malonate, acetate and glycerol. Acetate, first used by Smirnov in 1960, has the advantages of low cost and low rates of entry into central carbon metabolism. Thus it labels acyl groups, not head groups. The labeling of lipids in cultured Camelina embryos with respect to acetate concentration and time was investigated in the companion paper [Pollard et al., co-submitted]. By contrast, glycerol labels predominantly glyceryl head groups of lipids, though there is a small fraction of acyl group labeling [Bates et al., 2007 and 2009; Pollard et al., co-submitted]. When Camelina embryos are cultured at higher concentrations of exogenous glycerol $(>1 \mathrm{mM})$, the production of glycerol-3-phosphate for lipid synthesis from endogenous substrate via dihydroxyacetone phosphate reductase is effectively outcompeted by the action of glycerol kinase on exogenous glycerol [Pollard et al., cosubmitted].

For the analysis of labeled lipids and FAMEs produced from incubation of $\left[{ }^{14} \mathrm{C}\right]$ acetate with cultured embryos, some modifications to conventional TLC separation protocols were introduced. Base-catalyzed transmethylation results in recovery of $\geq 95 \%$ of radioactivity in the hexane phase, of which $>95 \%$ is in FAMEs. Not counting the very minor fatty acids $(\mathrm{C} 16: 3$, $\mathrm{C} 22: 2$ and C22:3) and pooling the minor positional isomers, in Camelina seed oil there are 13 forms of normal FAME. The longer chain fatty acids are more highly labeled by acetate through 
its preferential use for the cytosolic reactions of chain elongation [Ohlrogge et al., 1978; Pollard and Stumpf, 1980a, 1980b]. Due to incomplete resolution, analysis of labeling in each of the fatty acids cannot be simply accomplished by using both $\mathrm{C} 18$ reversed phase and conventional silver nitrate TLC analyses independently. One solution to this problem is to elute labeled bands and run each product on a second plate for the second dimension. To avoid the significant additional work involved in that approach, the silver nitrate chromatography system described by Wilson and Sargent (1992) was modified so that all the unsaturated FAMEs could be separated (Figure 6A). The C18 reversed phase separation can then be used to calculate the chain length distribution for the saturated FAMEs.

For lipid class analysis, PL separations used conventional silica TLC systems (Supplement Figure 4). However, modifications were made to TLC systems for neutral lipid (NL) analysis. Two-dimensional TLC showed that all lipid classes were resolved by a single NL separation plus a single PL TLC separation (Supplement Figure 4). Attention was paid to DAG and sterol separations as incomplete resolution was often observed using hexane-diethyl ether-acetic acid or toluene-ethyl acetate solvent systems. Sterols are disproportionately labeled from acetate relative to fatty acids (see below), yet DAG levels and labeling need to be accurately measured for flux analysis. Figure 6B shows two NL solvent systems applied to total lipid samples from [1${ }^{14} \mathrm{C}$ ]acetate or [U- $\left.{ }^{14} \mathrm{C}\right]$ glycerol substrates. The latter substrate does not label sterols. Tolueneethyl acetate solvent mixtures gave poor DAG versus sterol resolution but toluene-ethyl ether solvent mixtures gave consistent separation. The resolution of labeled [O]TAG from TAG is apparent for both solvent systems (Figure 6B). Other minor components (each $>0.5 \%$ of total label) are observed for acetate labeling but not for glycerol labeling, and are indicated by the asterisks in Figure 6. Labeled squalene, and 4-methyl and 4,4-diemethyl sterols have been noted in developing Cuphea seeds incubated with acetate [Singh et al., 1986]. The labeled compound annotated with a single asterisk in Figure 6 , panel B1, is too polar for either a cuticular hydrocarbon or squalene, but might be epoxy-squalene, while bands annotated ** in panels B1 and B2 may be 4-methyl and 4,4-diemethyl sterols.

Figure 6 shows that sterol labeling occurs from acetate but not from glycerol labeling. Labeled sterols are detected relatively late (> 12 hours), but provide information on the utilization of exogenous acetate by the cytosolic acetyl-CoA synthetase(s). Sterol biosynthesis requires the mevalonic acid pathway rather than the plastid 2-C-methyl-D-erythritol-4-phosphate pathway [Vranova et al., 2013], and thus uses cytosolic acetyl-CoA. This is also the precursor pool that provides malonyl-CoA for chain elongation catalyzed by FAE1-like condensing enzymes. Free sterols can be analyzed directly by GC-MS on a DB-5 or equivalent non-polar column and the isotopomer multiplet of the molecular ion observed. In developing embryos we observed only sitosterol and campesterol, in an approximately 65:35 ratio, as the dominant sterols. They were identified by both GC retention time relative to standards and their mass spectra. The major sterol of mature Camelina seed oil was reported to be sitosterol [Shukla et al., 2002]. The second most abundant sterol was campesterol, with lesser amounts of cholesterol, brassicasterol, stigmasterol and $\Delta 5$-avenasterol.

Figure 7 shows the molecular ion multiplet $(\mathrm{m} / \mathrm{z}=414)$ for sitosterol, from an assay using $\left[{ }^{13} \mathrm{C}_{2}\right]$ acetate as substrate. Campesterol was similarly labeled (data not shown). First, this confirms the identification of the labeled band as sterols. Consideration of the biosynthetic pathway to sitosterol reveals that 18 moles of acetate are required to produce one mole of sterol. Nine 9 moles of intact acetate moieties, 6 moles of isolated C(2) atoms and 3 moles of isolated $\mathrm{C}(1)$ atoms from acetate are incorporated, providing $27 \mathrm{C}$ atoms out of a total of $29 \mathrm{C}$ atoms. The lack of a pronounced $(M+2 n)$ repeating isotopomer pattern seen in Figure 7 is consistent with sitosterol containing 9 intact $\mathrm{C} 2$ units and 9 single carbon atoms from acetate. 
After subtracting the natural abundance isotopomers for unlabeled sitosterol, the remainder (ion current under the dotted line) contains ${ }^{13} \mathrm{C}_{2}$ to ${ }^{13} \mathrm{C}_{17}$ biosynthetically labeled species. The median lies between $(\mathrm{M}+7)$ and $(\mathrm{M}+8)(\mathrm{m} / \mathrm{z} 421$ and 422$)$, and if the value is $7.5{ }^{13} \mathrm{C}$ atoms, the contribution of exogenous substrate to biosynthesis is about $7.5 / 27=28 \%$. In these experiments, it is known that at saturating exogenous acetate concentrations plastid fatty acid synthesis utilizes $\sim 5 \%$ exogenous acetate [Pollard et al., co-submitted]. From this, it can be inferred that exogenous acetate enters the cytosolic acetyl-CoA pool about 5.6-fold more effectively than the plastid acetyl-CoA pool.

Although free sterols are a minor component $(\leq 0.35 \%)$ of Camelina seed oil [Shukla et al., 2002], they are preferentially labeled from acetate relative to fatty acids by a factor of about 6 . Thus, it is important in longer duration assays with acetate, where labeled DAG levels are relatively low, to have a clean TLC separation between sterol and DAG (Figure 6B).

Additionally, sterol isotopomer analysis will be a useful cross-check for $\left[{ }^{13} \mathrm{C}\right]$ acetate labeling experiments to assess relative contributions of exogenous acetate to plastid and cytosolic pools of acetyl-CoA. The sum of very-long-chain fatty acids (C20-C24) in 16 DPA embryos is 19.9 mole \% (Supplement Table 1). In the companion study [Pollard et al., co-submitted] it was shown that incorporation into total lipids saturated at $1-1.5 \mathrm{mM}$ acetate. The ${ }^{14} \mathrm{C}$ long-chain fatty acid labeling increased from 33-35\% at lower acetate concentrations to $43-45 \%$ at acetate $>2.5$ $\mathrm{mM}$. This difference likely reflects the differential competition and saturation kinetics for plastid and cytosolic acetyl-CoA synthesis to drive fatty acid synthesis and chain elongation, respectively. Methods for analysis of ${ }^{14} \mathrm{C}$-label distributions in unsaturated fatty acids by oxidative cleavage are rather tedious [Ohlrogge et al., 1978; Pollard and Stumpf, 1980a, 1980b; Bao et al., 1998]. This proposed short-cut via analysis of ${ }^{13} \mathrm{C}$ labeled products should prove adequate to correct acyl labeling to moles.

\section{3: Conclusions}

The developing seeds of $C$. sativa accumulate lipids at rates per unit fresh weight equivalent to oilseeds such as safflower or rapeseed. A striking feature of seed maturation in Camelina is a later stage surge and then abrupt cessation in linolenic acid deposition. The mechanisms underlying such kinetics remain to be determined, but once understood they may lead to a better understanding of the factors required to maximize polyunsaturated fatty acid accumulation in seeds. Minor polar fatty acids, namely 15,16-epoxy-and 15-hydroxyoctadecadienoic acids and homologous series of $\omega 7$-hydroxy-alk- $\omega 9$-enoic and $\omega 9 / 10$-hydroxyalkanoic acids, were identified. TLC and ESI-MS analysis of hydrogenated lipid fractions confirm that these epoxy- and hydroxy-fatty acids are indeed esterified to TAGs. For quantitative biochemical studies using cultured embryos, growth and lipid accumulation must parallel that of zygotic embryos. Cultured embryos at mid-maturation do indeed continue to accumulate lipid at rates equal and surpassing the rates seen for seeds in planta, and with a similar fatty acid composition. The cause of this 2-3 fold enhancement of lipid accumulation with extended culture is unknown, but may result in part by removal of nutrient limitation and/or hormonal factors in the embryo sac in the seed. In anticipation of developing quantitative flux maps of lipid biosynthesis using cultured Camelina embryos, attention has been paid to product identification, lipid class quantification and optimization of fatty acid and lipid separations. For example, the more polar TAG fractions noted above should be included in acyl flux accounting. FAME analysis to resolve all 13 individual fatty acids has been optimized, as has DAG separation from sterols. Furthermore, analysis of sterol ${ }^{13} \mathrm{C}$ labeling from acetate allows a shortcut to estimating the specific activity for acetate utilization in fatty acid synthesis and chain elongation. Together with the companion study [Pollard et al., co-submitted] on the utilization and limitations of exogenous acetate and glycerol for stable isotope $\left({ }^{13} \mathrm{C}\right)$ labeling of lipids in cultured embryos 
from oilseeds, the work should provide the methodological and analytical baseline to acyl lipid flux map construction through kinetic labeling experiments.

\section{4: Experimental}

\section{4:1 Plant materials}

Wild type $C$. sativa plants, var. Sunesson, were grown in a mixture of 3:1 potting soil (Sure-Mix, Michigan Grower's Products, Galesburg, MI) and vermiculite in a growth chamber at $60 \%$ relative humidity, $20^{\circ} \mathrm{C}, 16 / 8 \mathrm{hr}$ day/night cycle, and 150-160 $\mu \mathrm{E}$ light intensity (at the chamber floor). Plants were alternately watered with deionized $\mathrm{H}_{2} \mathrm{O}$ and half strength Hoagland's solution. Plants started to bolt after three weeks, with first flowers appearing at day 35-40. Flowers were tagged as petals opened. For seed development profiling, dissected seeds were immediately transferred to pre-weighed vials for fresh weight determination then dried at $85^{\circ} \mathrm{C}$ for $4-6$ hours in a convection oven for dry weight measurement. For lipid analysis at mid-maturation, embryos (16 DPA) were dissected and immediately frozen in liquid $\mathrm{N}_{2}$. Embryos were stored at $-70^{\circ} \mathrm{C}$ until use. For embryo culture, siliques were harvested into $20 \%$ bleach then rinsed thoroughly prior to seed removal and embryo dissection.

\section{4:2 Embryo culture and incubations}

Embryos at 15 DPA unless otherwise stated were aseptically dissected from developing $C$. sativa seeds and transferred to 6 well culture plates. Each well contained culture medium (1.0 $\mathrm{ml})$, with 5 embryos per well. The plate lid was attached with Parafilm and the plate incubated at $20^{\circ} \mathrm{C}$ with gentle rocking $(0.2-0.3 \mathrm{~Hz})$ in green light at $10-12 \mu \mathrm{E}$. This light regime approximates that reaching the seed in the silique. The culture medium reflects the composition of the embryo sac fluid (Cocuron, J.-C. and Shachar-Hill,Y., unpublished results). It contained alanine (4 mM), glutamine $(8 \mathrm{mM})$, glucose $(130 \mathrm{mM})$ and sucrose $(12 \mathrm{mM})$ as macronutrients. It also included Gamborg's vitamins, and mineral micronutrients as used for $B$. napus embryo culture [Schwender and Ohlrogge, 2002] but minus the ammonium and potassium nitrates, so that the only $\mathrm{N}$ source is from amino acids. pH was maintained at 6.3 with $20 \mathrm{mM} \mathrm{HEPES}$, while $20 \%$ $(\mathrm{w} / \mathrm{v})$ polyethylene 4000 provided the osmoticum. To measure embryo growth, embryos were removed at defined times, rinsed in $\mathrm{DI}_{2} \mathrm{O}$, blotted dry and transferred to pre-weighed vials for weighing. After obtaining fresh weights, the embryos were lyophilized to give dry weight. For lipid labeling assays, after one day in culture, an additional $0.5 \mathrm{ml}$ of medium containing either $2.5 \mathrm{mM} \mathrm{NaOAc}$ plus $\left[1{ }^{-14} \mathrm{C}\right] \mathrm{NaOAc}$ (Perkin Elmer NEC084H001, $2.101 \mathrm{MBq} / \mathrm{mol}, 0.2775 \mathrm{MBq}$ ) or $0.3 \mathrm{mM}$ glycerol plus [U- ${ }^{14} \mathrm{C}$ ]glycerol (Perkin Elmer NEC441X, 5.217 MBq/mol, $0.235 \mathrm{MBq}$ ) was added per well and embryos were incubated for the allotted time, removed and washed prior to hot $\mathrm{PrOH}$ quenching and lipid extraction.

\section{4:3 Lipid extractions}

Lipids were extracted with hexane-IPrOH according to the method of Hara and Radin (1978). For the seed development profile, dried seed was ground in a homogenizer and directly extracted with hexane-iPrOH, with internal standards tripentadecanoin and diheptadecanoylphosphatidylcholine added during the extraction. For lipid extractions with either freeze-dried or frozen embryos or washed fresh embryos from culture, embryos were heated at $80-85^{\circ} \mathrm{C}$ in iPrOH for 10-15 minutes to inactivate lipases prior to lipid extraction. Tripentadecanoin was added during extraction as an internal standard for fatty acid quantification. After extraction, the 
total lipids were stored in toluene at $-20^{\circ} \mathrm{C}$ until aliquots were withdrawn for the variety of lipid analyses described below. The lipid masses recovered from the seed development profile samples were sufficient for gravimetric determinations. Estimation of total fatty acids by transmethylation and GC analysis produced lipid mass values that were on average $88 \pm 5 \%$ of the gravimetric analysis of lipid content.

\section{4:4 General methods}

Chlorophyll content was determined in heptane solutions of lipid extracts using absorption at 645 and $663 \mathrm{~nm}$, with slightly modified extinction coefficients to account for the difference in solvent from the original method [Arnon, 1949]. TLC plates with ${ }^{14} \mathrm{C}$ labeled samples were subject to autoradiography using Kodak Phosphor Screens GP. After exposure, the screens were scanned using the Bio-Rad PMI FX phosphoimager and radioactivity bands quantified using Bio-Rad Quantity One basic software.

\section{4:5 Fatty acid analyses}

Transmethylation of total lipids or lipid fractions from preparative TLC was accomplished using the biphasic $\mathrm{KOH}-\mathrm{MeOH}$-heptane protocol of Ichihara et al. (1996). This method uses mild conditions, which is desirable for highly unsaturated samples, and has the added advantage of leaving epoxide groups intact. FAMEs were analyzed by GC with FID detection (Agilent 6890N, split injection at $250^{\circ} \mathrm{C}$, oven temperature ramp from $140^{\circ} \mathrm{C}$ to $230^{\circ} \mathrm{C}$ at $5^{\circ} \mathrm{C} / \mathrm{min}$ ) on a DB-23 capillary column ( $30 \mathrm{~m} \times 0.25 \mathrm{~mm}$ id, $0.25 \mu$ film thickness). Peak areas were corrected for $\mathrm{C}(\mathrm{H})$ response [Bannon et al., 1986; Craske and Bannon, 1987]. Labeled FAMEs were separated by a combination of $\mathrm{AgNO}_{3}$ and reversed phase TLC. K6 TLC plates were impregnated with $7.5 \%$ (w/v) $\mathrm{AgNO}_{3}$ solution in $\mathrm{CH}_{3} \mathrm{CN}$. After drying and sample application, they were developed halfway in toluene- $\mathrm{CH}_{3} \mathrm{CN}$ (98:2), quickly air-dried and developed fully in toluene. $\mathrm{KC} 18 \mathrm{~F}$ reversed phase TLC plates were developed using $\mathrm{MeOH}-\mathrm{CH}_{3} \mathrm{CN}-\mathrm{H}_{2} \mathrm{O}$ (65:35:0.5). Figure $6 \mathrm{~A}$ shows a representative example of these separations.

For identification of fatty acids by mass spectrometry, an Agilent 6850-5975MSD GC-MS system was used. FAMEs were separated on a DB-5 capillary column $(30 \mathrm{~m} \times 0.25 \mathrm{~mm}$ id, 0.25 film thickness) using splitless injection at $310^{\circ} \mathrm{C}$ and a temperature ramp from $120^{\circ} \mathrm{C}$ to $310^{\circ} \mathrm{C}$ at $10^{\circ} \mathrm{C} / \mathrm{min}$, with eluant analysis by electron impact mass spectrometry. FAME samples for GC and GC-MS analysis containing hydroxy-FAMEs were first silylated by heating at $110^{\circ} \mathrm{C}$ for $10^{-}$ 20 minutes in BSTFA-pyridine (1:1) solution. After reaction, samples were evaporated to dryness under $\mathrm{N}_{2}$ gas and resuspended in heptane.

A sample of Euphorbia lagascae seed oil was used as a standard for epoxy-TAGs [Kleiman et al., 1965] and to generate both methyl 12,13-cis-epoxyoctadec-9-cis-enoate (vernolate) and methyl 12,13-threo-dihydroxy-octadec-9-cis-enoate standards. The latter was prepared by first heating the oil in $\mathrm{AcOH}$ overnight at $110-115^{\circ} \mathrm{C}$ to open the epoxide ring prior to transmethylation [Gunstone and Morris, 1959]. Samples of Lesquerella fendleri and Ricinus communis seed oils provided hydroxy-TAG and hydroxy-FAME standards.

\section{4:6 Lipid analyses}

Several silica TLC systems were used for neutral lipid (NL) and polar lipid (PL) separations (See Figure 6B and Supplement Figure 4). For preparative TLC analysis of endogenous lipids, plates were developed in solvents containing a trace $(\sim 0.001 \% \mathrm{w} / \mathrm{v})$ butylated hydroxytoluene, and after chromatography, lipid bands were visualized by spraying with primuline $(0.01 \% \mathrm{w} / \mathrm{v})$ in 
acetone- $\mathrm{H}_{2} \mathrm{O}(4: 1)$, briefly air drying and viewing the plate under long wavelength UV light (355 $\mathrm{nm}$ ). For preparative work, NL were separated using developments of silica TLC plates with toluene- $\mathrm{Et}_{2} \mathrm{O}$ (7:3). PL were separated using $\left(\mathrm{NH}_{4}\right)_{2} \mathrm{SO}_{4}$-impregnated, heated activated TLC plates, developed with acetone-toluene- $\mathrm{H}_{2} \mathrm{O}(91: 30: 8)$ [Khan and Williams, 1977]. NL were eluted from the silica with $\mathrm{CHCl}_{3}-\mathrm{MeOH}(2: 1)$, $\mathrm{PL}$ with $\mathrm{CHCl}_{3}-\mathrm{MeOH}-\mathrm{H}_{2} \mathrm{O}$ (5:5:1). To correct for incomplete lipid recovery, an external standard, either ditetradecanoin or ditetradecanoyl phosphatidylethanolamine, was added to the silica prior to elution.

For hydrogenation, lipid samples were stirred in heptane-EtOAc $(3 \mathrm{ml}, 9: 1)$ at slightly greater than atmospheric pressure of hydrogen in the presence of platinum (VI) oxide catalyst (ca. 2 $\mathrm{mg}$ ). Complete reaction was achieved within 2 hours at room temperature, after which the catalyst was decanted. The reaction product was typically further fractionated by TLC and samples analyzed by ESI-MS. For mass spectrometry, lipid or hydrogenated lipid samples were dissolved in $\mathrm{PrOH}$, typically at $10 \mu \mathrm{g} / \mathrm{ml}$. ESI-MS in positive ion mode was performed by direct infusion with a Shimadzu (Columbia, MD) SIL-5000 autosampler into a Waters (Milford, MA) Quattro micro mass spectrometer. Sample solutions $(10 \mu \mathrm{l})$ were introduced to the electrospray source by flow injection into a 97:3 $\mathrm{PrOH}: 10 \mathrm{mM}$ aqueous $\mathrm{NH}_{4} \mathrm{OAc}$ buffer flowing at $0.1 \mathrm{ml} / \mathrm{min}$. The capillary, extractor, and cone voltages were $3.2 \mathrm{kV}, 2.0 \mathrm{~V}$, and $25 \mathrm{~V}$ respectively. The source and desolvation temperatures were 110 and $350{ }^{\circ} \mathrm{C}$ respectively; the desolvation gas flow rate was $400 \mathrm{l} / \mathrm{hr}$. Mass spectra were collected for $2 \mathrm{~min}$; the $\mathrm{m} / \mathrm{z}$ range scanned in the MS measurements was from 600 to 1100 (1 sec/scan). Mass spectra data were acquired with MassLynx 4.0 software; [TAG-NH $\left.{ }^{4}\right]^{+}$ion peaks were smoothed and integrated using QuanLynx software.

\section{Acknowledgements}

The authors gratefully acknowledge: Lisa Carey for details of $C$. sativa embryo culturing conditions developed by herself and Jean-Christophe Cocuron in this laboratory; Dr. Dan Jones and Lijun Chen of the Michigan State University RTSF Mass Spectrometry \& Metabolomics Core for ESI-MS technical support and instrumentation; and the Center for Advanced Biofuels Systems, an Energy Frontier Research Center funded by the US Department of Energy, Office of Science, and Office of Basic Energy Sciences under award number DE-SC0001295 and the Great Lakes Bioenergy Research Center (DOE BER Office of Science DE-FC02-07ER64494) for financial support. 


\section{References}

Abramovic, H., Abram, V., 2005. Physico-chemical properties, composition and oxidative stability of Camelina sativa oil. Food Technol. Biotechnol. 43, 63-70.

Arnon, D.I ., 1949. Copper enzymes in isolated chloroplasts. Polyphenol oxidase in Beta vulgaris. Plant Physiol. 24, 1-15.

Bannon, C.D., Craske, J.D., Hilllker, A.E., 1986. Analysis of fatty acid methyl esters with high accuracy and reliability: $\mathrm{V}$. Validation of theoretical relative response factors of unsaturated esters in the flame ionization detector. J. Amer. Oil Chem. Soc 63, 105-110.

Bates, P.D., Browse, J., 2012. The significance of different diacylgycerol synthesis pathways on plant oil composition and bioengineering. Front. Plant Sci. 3,147.

Bates, P.D., Durrett, T.P., Ohlrogge, J.B., Pollard, M., 2009. Analysis of acyl fluxes through multiple pathways of triacylglycerol synthesis in developing soybean embryos. Plant Physiol. $150,55-72$.

Bates, P.D., Ohlrogge, J.B., Pollard, M., 2007. Incorporation of newly-synthesized faty acids into cytosolic glycerolipids in pea leaves occurs via acyl editing. J. Biol. Chem. 282, 3120631216.

Bates, P.D., Stymne, S., Ohlrogge, J., 2013. Biochemical pathways in seed oil synthesis. Curr. Opin. Plant Biol. 16, 358-364.

Baud, S., Boutin, J.-P., Miquel, M., Lepiniec, L., Rochat ,C., 2002. An integrated overview of seed development in Arabidopsis thaliana ecotype WS. Plant Physiol. Biochem. 40, 151-160.

Brieskorn, C.H., Kabelitz, L., 1971. Hydroxyfettsauren aus dem cutin des blattes von Rosmarinus officinalis. Phytochemistry 10, 3195-3204.

Broun, P., Boddupalli, S., Somerville, C., 1998. A bifunctional oleate 12-hydroxylase: desaturase from Lesquerella fendleri. Plant J. 13, 201-210.

Budin, J.T., Breene, W.M., Putnam, D.H., 1995. Some compositional properties of Camelina (Camelina sativa L. Crantz) seeds and oils. J. Amer. Oil Chem. Soc. 72, 309-315.

Cahoon, E.B., Shockey, J.M., Dietrich, C.R., Gidda, S.K., Mullen, R.T., Dyer, J.M., 2007. Engineering oilseeds for sustainable production of industrial and nutritional feedstocks: solving bottlenecks in fatty acid flux. Curr. Opin. Plant Biol. 10, 236-244.

Clemente, T.E., Cahoon, E.B., 2010. Soybean Oil: Genetic approaches for modification of functionality and total content. Plant Physiol. 151, 1030-1040.

Craske, J.D., Bannon, C.D., 1987. Gas liquid chromatography analysis of the fatty acid composition of fats and oils: A total system for high accuracy. J. Amer. Oil Chem. Soc. 64, 1413-1417.

Frohlich, A., Rice, B., 2005. Evaluation of Camelina sativa oil as a feedstock for biodiesel production. Ind. Crops Prod. 21, 25-31. 
Girke, T., Todd, J., Ruuska, S., White, J., Benning, C., Ohlrogge, J., 2000. Microarray analysis of developing Arabidopsis seeds. Plant Physiol. 124, 1570-1581.

Gunstone, F.D., Morris, L.J., 1959. Fatty acids. Part VI. The oxygenated acid present in Camelina sativa (Crantz.) seed oil. J. Chem. Soc. 2127-2132.

Hamberg, M., Hamberg, H. (1996) 15(R)-Hydroxylinoleic acid, an oxylipin from oat seeds. Phytochemistry 42, 729-732.

Hara, A., Radin, N.S., 1978. Lipid extraction of tissues with a low-toxicity solvent. Anal. Biochem. 90, 420-426.

Holloway, P.J., 1984. Cutins and suberins, the polymeric plant lipids. In: Mangold, H.K., Zweig, G., Sherma, J. (Eds.), CRC Handbook of Chromatography, Lipids, vol. 1. CRC Press, Boca Raton, pp. 321-345.

Horn, P.J., Silva, J.E., Anderson, D., Fuchs, J., Borisjuk, L., Nazarenus, T.J., Shulaev, V., Cahoon, E.B., Chapman, K.D., 2013. Imaging heterogeneity of membrane and storage lipids in transgenic Camelina sativa seeds with altered fatty acid profiles. Plant J. 76, 138-150.

Ichihara, K., Shibahara, A., Yamamoto, K., Nakayama, T., 1996. An improved method for rapid analysis of the fatty acids of glycerolipids. Lipids 31, 535-539.

Kang, J., Snapp, A.R., Lu, C., 2011. Identification of three genes encoding microsomal oleate desaturases (FAD2) from the oilseed crop Camelina sativa. Plant Physiol. Biochem. 49, 223229.

Kleiman, R., Smith, C.R. Jr., Yates, S.G., 1965. Search for new industrial oils: fifty-eight Euphorbiaceae oils, including one rich in vernolic acid. J Amer. Oil Chem. Soc. 42, 169-172.

Khan, M.U., Williams, J.P., 1977. Improved thin-layer chromatographic method for the separation of major phospholipids and glycolipids from plant lipid extracts and phosphatidyl glycerol and bis(monoacylglycerol)phosphate from animal lipid extracts. J. Chromatogr. 140, 179-185.

Li, Y., Beisson, F., Pollard, M., Ohlrogge, J., 2006. Oil content of Arabidopsis seeds: The influence of seed anatomy, light and plant-to-plant variation. Phytochemistry 67, 904-915.

Liang, C., Liu, X., Yiu, S.-M., Lim, B.L., 2013. De novo assembly and characterization of Camelina sativa transcriptome by paired-end sequencing. BMC Genomics 14, 146-156.

Liu, J., Rice, A., McGlew, K., Shaw, V., Park, H., Clemente, T., Pollard, M., Ohlrogge, J., Durrett, T., 2015. Metabolic engineering of oilseed crops to produce high levels of novel acetyl glyceride oils with reduced viscosity, freezing point and calorific value. Plant Biotechnol. J. DOI: $10.1111 /$ pbi.12325

Lu, C.-F., Kang, J.-L., 2008. Generation of transgenic plants of a potential oilseed crop Camelina sativa by Agrobacterium-mediated transformation. Plant Cell Rep. 27, 273-278. 
Mansour, M.P., Shrestha, P., Belide, S., Petrie, J.R., Nichols, P.D., Singh, S.P., 2014. Characterization of oilseed lipids from "DHA-producing Camelina sativa": a new transformed land plant containing long-chain omega-3 oils. Nutrients 6, 776-789.

Millar, A.A., Kunst, L., 1997. Very-long-chain fatty acid biosynthesis is controlled through the expression and specificity of the condensing enzyme. Plant J. 12, 121-131.

Mongrand, S., Bessoule, J.-J., Cabantous, F., Cassagne, C., 1998. The C16:3/C18:3 fatty acid balance in photosynthetic tissues from 468 plant species. Phytochemistry 49, 1049-1064.

Moser, B.R., Vaughn, S.F., 2010. Evaluation of alkyl esters from Camelina sativa oil as biodiesel and as blend components in ultra low-sulfur diesel fuel. Bioresour. Technol. 101, 646653.

Mudalkar, S., Golla, R., Ghatty, S., Ramachandra, A., 2014. De novo transcriptome analysis of an imminent biofuel crop, Camelina sativa L. using Illumina GAIIX sequencing platform and identification of SSR markers. Plant Mol. Biol. 84, 159-171.

Napier, J.A., Haslam, R.P., Beaudoin, F., Cahoon, E.B., 2014. Understanding and manipulating plant lipid composition: Metabolic engineering leads the way. Curr. Opin. Plant Biol. 19, 68-75.

Ohlrogge, J.B., Pollard, M.R., Stumpf, J.B., 1978. Studies on biosynthesis of waxes by developing Jojoba seed tissue. Lipids 13, 203-210.

Plattner, R.D., Payne-Wahl, K., Tjarks, L.W., Kleiman, R., 1979. Hydroxy acids and estolide triacylglycerides of Heliophila amplexicaulis L.f. seed oil. Lipids 14, 576-579.

Pollard, M., Delamarter, D., Martin, T.M., Shachar-Hill, Y., 2015. Lipid labeling from acetate or glycerol in cultured embryos of Camelina sativa seeds: A tale of two substrates. Submitted together with this manuscript to Phytochemistry.

Pollard, M.R., Stumpf, P.K., 1980. Biosynthesis of C20 and C22 fatty acids by developing seeds of Limnanthes alba; chain elongation and $\Delta 5$ desaturation. Plant Physiol. 66, 649-655.

Pollard, M.R., Stumpf, P.K., 1980. Long chain (C20 and C22) fatty acid biosynthesis in developing seeds of Tropaeolum majus; an in vivo study. Plant Physiol. 66, 641-648.

Rodriguez-Rodriguez, M.F., Sanchez-Garcia, A., Salas, J.J., Garces, R., Martinez-Force, E., 2013. Characterization of the morphological changes and fatty acid profile of developing Camelina sativa seeds. Ind. Crops Prod. 50, 673-679.

Ruuska, S., Girke, T., Benning, C., Ohlrogge, J., 2002. Contrapuntal networks of gene expression during Arabidopsis seed filling. Plant Cell 14, 1191-1206.

Schwender, J., Ohlrogge, J.B., 2002. Probing in vivo metabolism by stable isotope labeling of storage lipids and proteins in developing Brassica napus embryos. Plant Physiology, 130, 347-361.

Shukla, V.K.S., Dutta, P.C., Artz, W.E., 2002. Camelina oil and its unusual cholesterol content. J. Amer. Oil Chem. Soc. 79, 965-969. 
Singh, S.S., Nee, T.Y., Pollard, M.R., 1986. Acetate and mevalonate labeling studies with developing Cuphea lutea seeds. Lipids 21, 143-149.

Smirnov, B.P., 1960. The biosynthesis of higher fatty acids from acetate in isolated chloroplasts of Spinicia oleacera. Biokhimiya 25, 545-555.

Vranova, E., Coman, D., Gruissem, W., 2013. Network analysis of the MVA and MEP pathways for isoprenoid synthesis. Annu. Rev. Plant Biol. 64, 665-700.

Wilson, R., Sargent, J.R., 1992. High-resolution separation of polyunsaturated fatty acids by argentation thin-layer chromatography. J. Chromatogr. 623, 403-407.

Zubr, J., Matthus, B., 2002. Effects of growth conditions on fatty acids and tocopherols in Camelina sativa oil. Ind. Crops Prod. 15, 155-162. 


\section{Figure Legends}

Figure 1: Lipid ( $\square)$, dry matter $(\circ)$, moisture $(\diamond)$ and chlorophyll $(+)$ accumulation by $C$. sativa seeds during development. Individual data points are shown, with each data point representing 150-300 seed sample harvested from multiple plants. Chlorophyll is expressed as $\mathrm{ng} / \mathrm{seed}$ rather than $\mu \mathrm{g} / \mathrm{seed}$.

Figure 2: Accumulation of the major individual fatty acids in $C$. sativa seeds during development. Panel A shows the masses, with both average values (larger and/or filled symbols) and the individual determinations ( $n=2$ to 5 , smaller, open symbols). Panel B shows the relative rates of accumulation (arbitrary units). The data points are directly derived from the average slopes shown in panel $A$.

Figure 3: GC(FID) analysis on DB-23 column of epoxy and hydroxy fatty acid methyl esters from TAG fractions isolated by preparative TLC from $C$. sativa seed lipids. Panel A: FAMEs from total lipids of mature $C$. sativa seeds, with identification of the major components. Panel B: FAMEs from an epoxy-TAG fraction ("EPOXY-TAG" in text). The major epoxy FAME, 15,16-epoxy C18:2 is shown. A methyl vernolate standard (12,13-epoxy C18:1) was also run to give an accurate retention time. There is a negligible amount of this fatty acid present. Panel $\mathrm{C}$ : Trimethylsilylated FAMES from the hydroxy-TAG fraction ("EPOXY-TAGl" in text). Identified hydroxy FAMEs are: 1, methyl ricinoleate (methyl 12-hydroxyoctadec-9-enoate); 2, methyl densipolate (methyl 12-hydroxyoctadec-9,15-dienoate); 3, methyl 15-hydroxyoctadecadienoate; 4, methyl 12-hydroxyeicosanoate (major isomer); 5 , methyl lesquerolate (methyl 14hydroxyeicos-11-enoate); 6, methyl 13-hydroxydocosanoate (major isomer); and 7, methyl 16hydroxydocos-13-enoate). 8 is 15,16-epoxyoctadeca-9,12-dienoate.

Figure 4: ESI-MS analysis of hydrogenated TAGs, epoxy-TAGs and mixed hydroxy- and epoxyTAG TLC-purified fractions from the oil of mature $C$. sativa seeds. Base peak $\mathrm{m} / \mathrm{z}$ values are given, while structure assignments are boxed. Panel A: The non-polar TAG fraction shows the major homologous series of C52 to C58 TAG $\left(\mathrm{M}+\mathrm{NH}_{4}\right)^{+}$adducts, separated by $\Delta=28$ amu, where $\mathrm{C}$ indicates the number of acyl carbon atoms. Minor adduct series for $(\mathrm{M}+\mathrm{Na})^{+}(\Delta=5$ amu) and at $\Delta=13,14$ amu are apparent. Panel B: Hydrogenated epoxy-TAG fraction ("EPOXY-TAG" in text). In this fraction the $\mathrm{m} / \mathrm{z}$ values for the major homologous series are increased by $\Delta=14 \mathrm{amu}$, when compared to the series shown in panel $A$. This is consistent with the TAGs containing a single epoxide group. Panel $\mathrm{C}$ : Hydrogenated mixed hydroxy- and epoxy-TAG fractions ("EPOXY-TAGl" in text). This TLC band is rather diffuse. The major components seen in the epoxy-TAG rich fraction $(\mathrm{m} / \mathrm{z}=894.8,922.8,950.8$ and 978.9, Panel B) are still present, but the homologous series at 2 amu higher is now dominant. This represents TAGs containing a hydroxyl group. An additional series at $\mathrm{m} / \mathrm{z}=910.8,938.8,966.8$ and 994.9 is most likely TAGs containing both epoxy and hydroxy groups.

Figure 5: Culture of 15 days post anthesis (DPA) embryos from C. sativa. Panel A shows moisture $(X)$, dry mass $(\boldsymbol{\bullet})$ and total fatty acid $(\bullet)$ accumulations (average values). Triplicate individual determinations are also shown (smaller, open symbols). Average values for zygotic embryos harvested from plants at 12-16 DPA are included in order to assess the progression prior to culture (dotted lines). Panel B superimposes average values for dry weight $(\mathbf{\square}, \square)$ and lipid weight $(\bullet, \circ)$ for the cultured embryos (filled symbols) with that for developing seeds (open symbols). Panel $\mathrm{C}$ shows the accumulation of major individual fatty acids in both cultured embryos and also zygotic embryos harvested from plants at 14 and 16 DPA. Again average values are the larger and/or closed symbols, while the triplicate individual determinations are smaller, open symbols. 
Figure 6: TLC analyses of $\left[{ }^{14} \mathrm{C}\right]$ fatty acid methyl esters (FAMEs) and $\left[{ }^{14} \mathrm{C}\right]$ lipids. Panel A1-A3 shows multiple analyses of $\left[{ }^{14} \mathrm{C}\right] \mathrm{FAMEs}$ from total lipids. The 48 hour incubation allows substantial production of $\left[{ }^{14} \mathrm{C}\right]$ linolenic acid. Analysis by $\mathrm{AgNO}_{3} \mathrm{TLC}(7.5 \% \mathrm{w} / \mathrm{w}$ in $\mathrm{K} 6$ silica) developed with hexane/ $\mathrm{Et}_{2} \mathrm{O}(1: 1 \mathrm{v} / \mathrm{v})$ shows separation based only on number of double bonds, with tailing of the trienoic FAME (A1). Instead, developing half way with toluene- $\mathrm{CH}_{3} \mathrm{CN}(98: 2)$ then fully with toluene greatly improves separation of unsaturated FAME (A2). A standard C18 reversed-phase TLC analysis using $\mathrm{MeOH}-\mathrm{CH}_{3} \mathrm{CN}-\mathrm{H}_{2} \mathrm{O}$ (65:35:0.5) resolves the $\left[{ }^{14} \mathrm{C}\right] \mathrm{FAME}$ mixture into a six discrete bands (A3). Running TLC systems A2 and A3 independently on the same FAME sample allows determination of the full $\left[{ }^{14} \mathrm{C}\right] \mathrm{FAME}$ composition.

Panel B1,B2 shows K6 silica neutral lipid TLC analyses from embryos cultured with [1${ }^{14} \mathrm{C}$ ]acetate for 48 hours (Ac) or [U- ${ }^{14} \mathrm{C}$ ] glycerol for 8 hours (Gly) using single developments of either toluene:EtOAc $(86: 14 \mathrm{v} / \mathrm{v})(\mathrm{B} 1)$ or toluene: $\mathrm{Et}_{2} \mathrm{O}(7: 3 \mathrm{v} / \mathrm{v})(\mathrm{B} 2)$. The acetate incubation was run for 48 hours to allow good sterol and [O]TAG labeling. Abbreviations:- TAG: triacylglycerols, [O]TAG: triacylglycerols where at least one fatty acid contains an epoxy or hydroxy functional group, DAG: Diacylglycerol, PL: total polar lipids. Minor bands marked with asterisks are identified, but may represent intermediates in the sterol biosynthesis pathway.

Figure 7: Analysis of $\left[{ }^{13} \mathrm{C}_{2}\right]$ acetate-labeled sitosterol. Cultured Camelina embryos were labeled with $2.6 \mathrm{mM}\left[{ }^{13} \mathrm{C}_{2}\right.$ ] acetate for 3 days. Labeled sterols were recovered by TLC and analyzed without further derivatization by GC-MS(EI) using a DB-5 GC column with a temperature ramp to $320^{\circ} \mathrm{C}$. The molecular ion multiplet for sitosterol is shown, with the Peak Intensity axis truncated to allow a better view of the isotopomer multiplet. The ion current for the base peak at $\mathrm{m} / \mathrm{z}=414$ is 55000 units. The dotted red line shows the ${ }^{13} \mathrm{C}$ multiplet after subtraction of the base peak natural abundance profile. ${ }^{13} \mathrm{C}$ Labeled sitosterol is $29.6 \%$ of the total multiplet ion current. The median $\mathrm{m} / \mathrm{z}$ value for the ${ }^{13} \mathrm{C}$ labeled multiplet lies between $\mathrm{m} / \mathrm{z}=421$ and 422 $(\mathrm{M}+7.5)$. 
Fig. 1

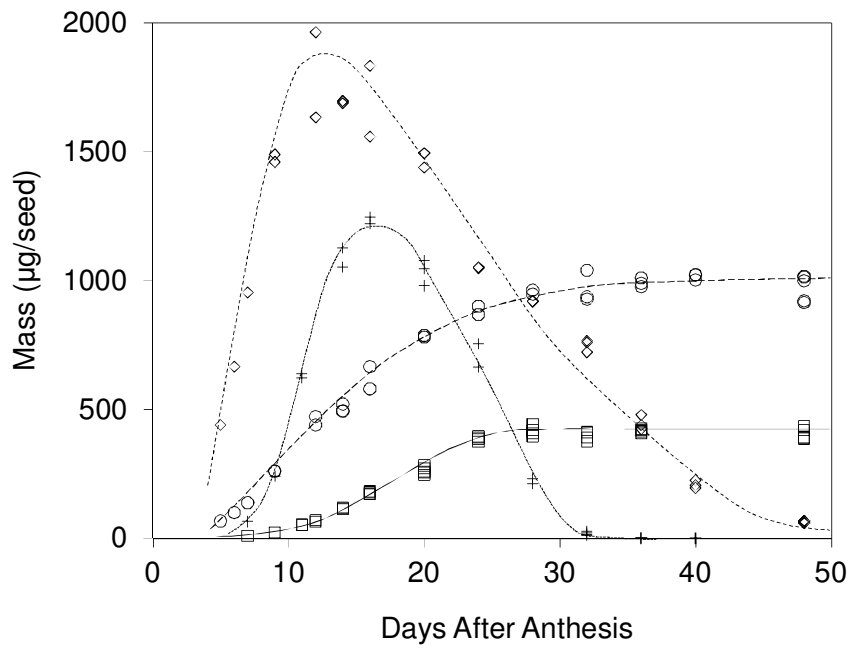


Figure 2
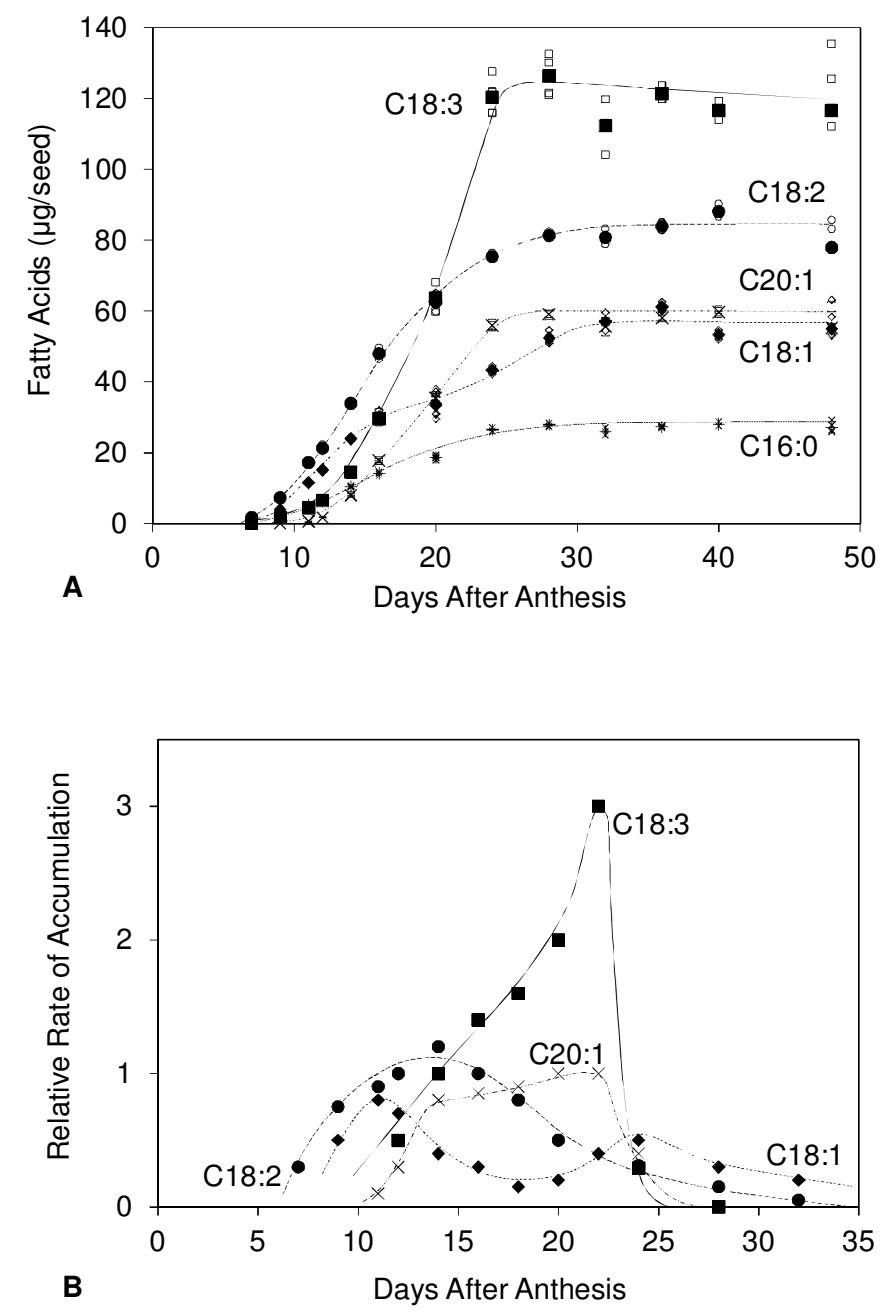
Figure 3
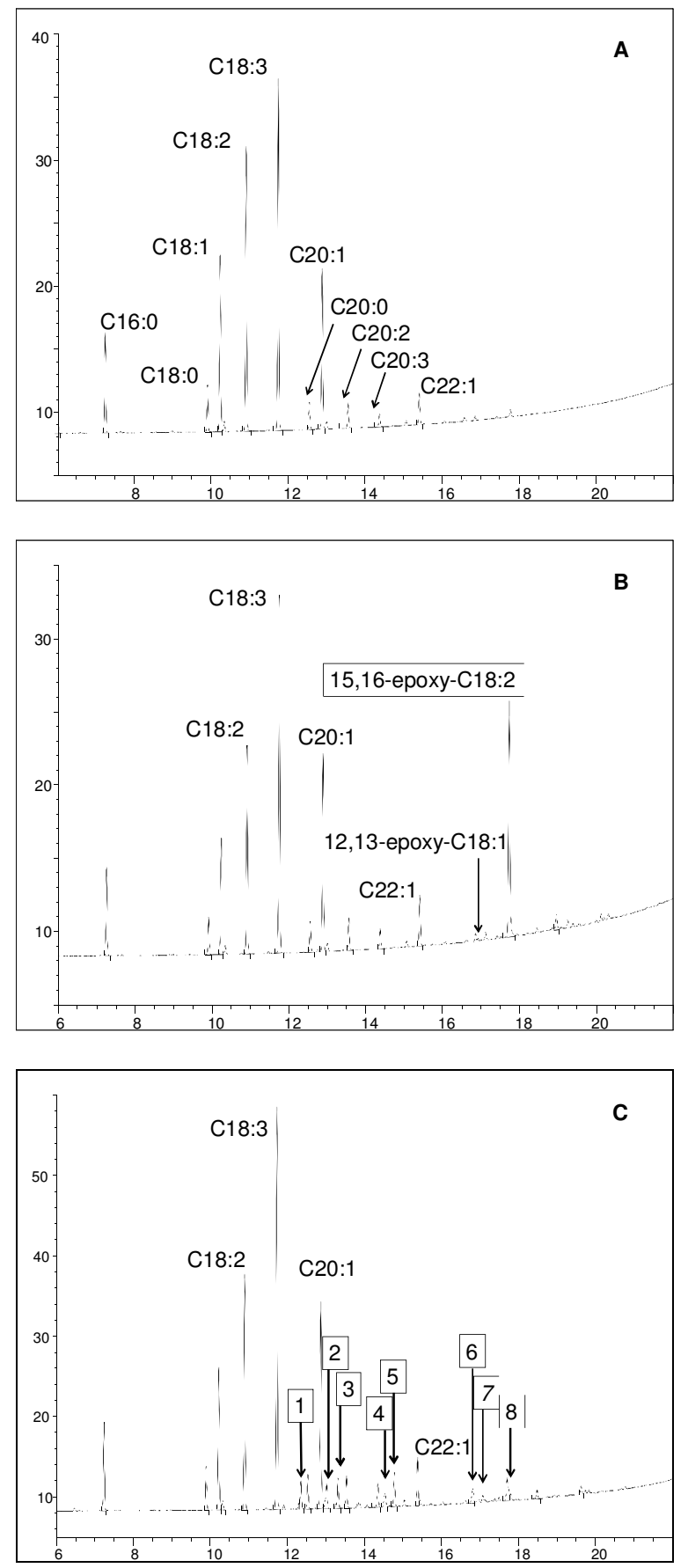
Figure 4:

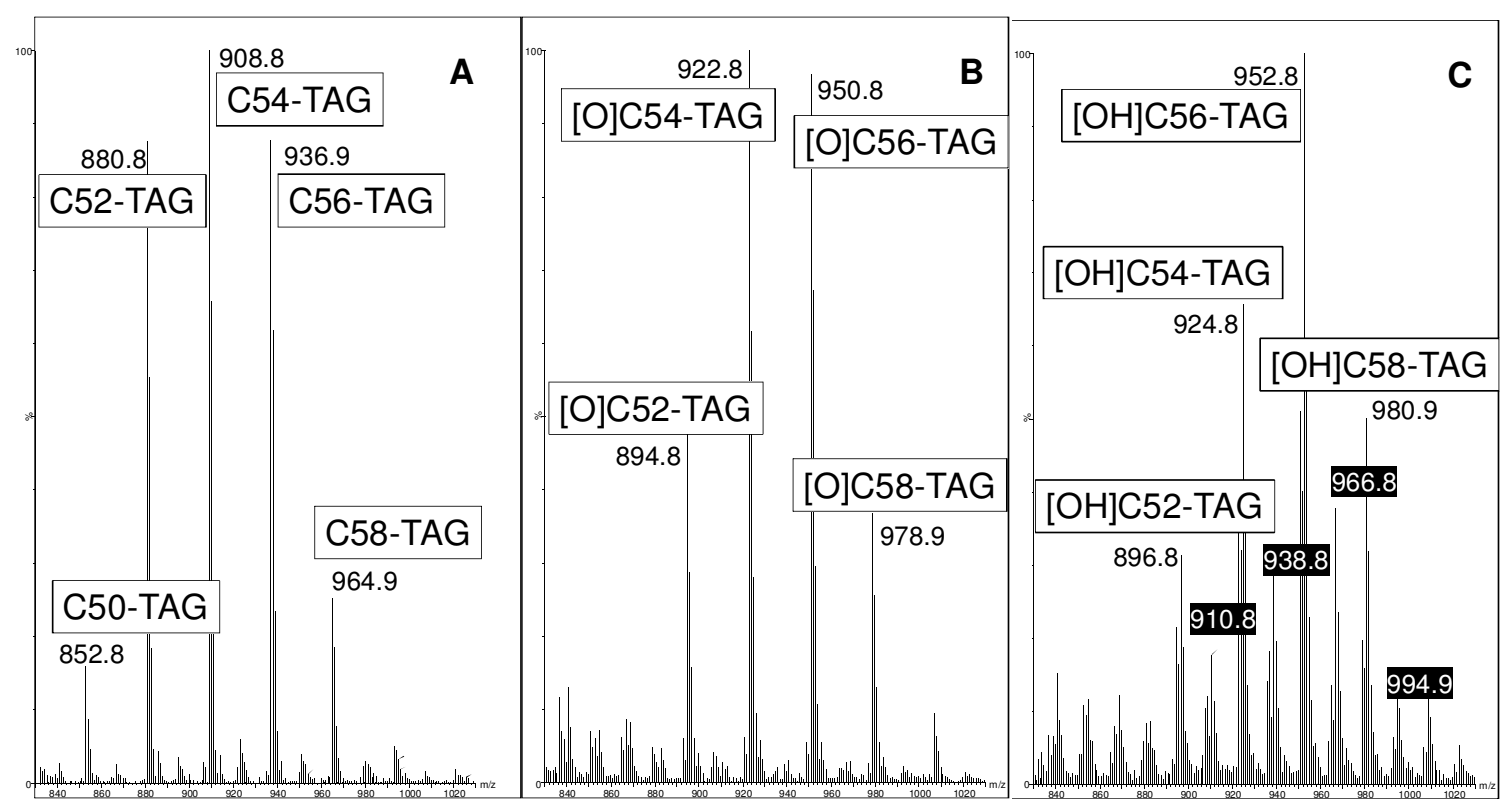


Figure 5
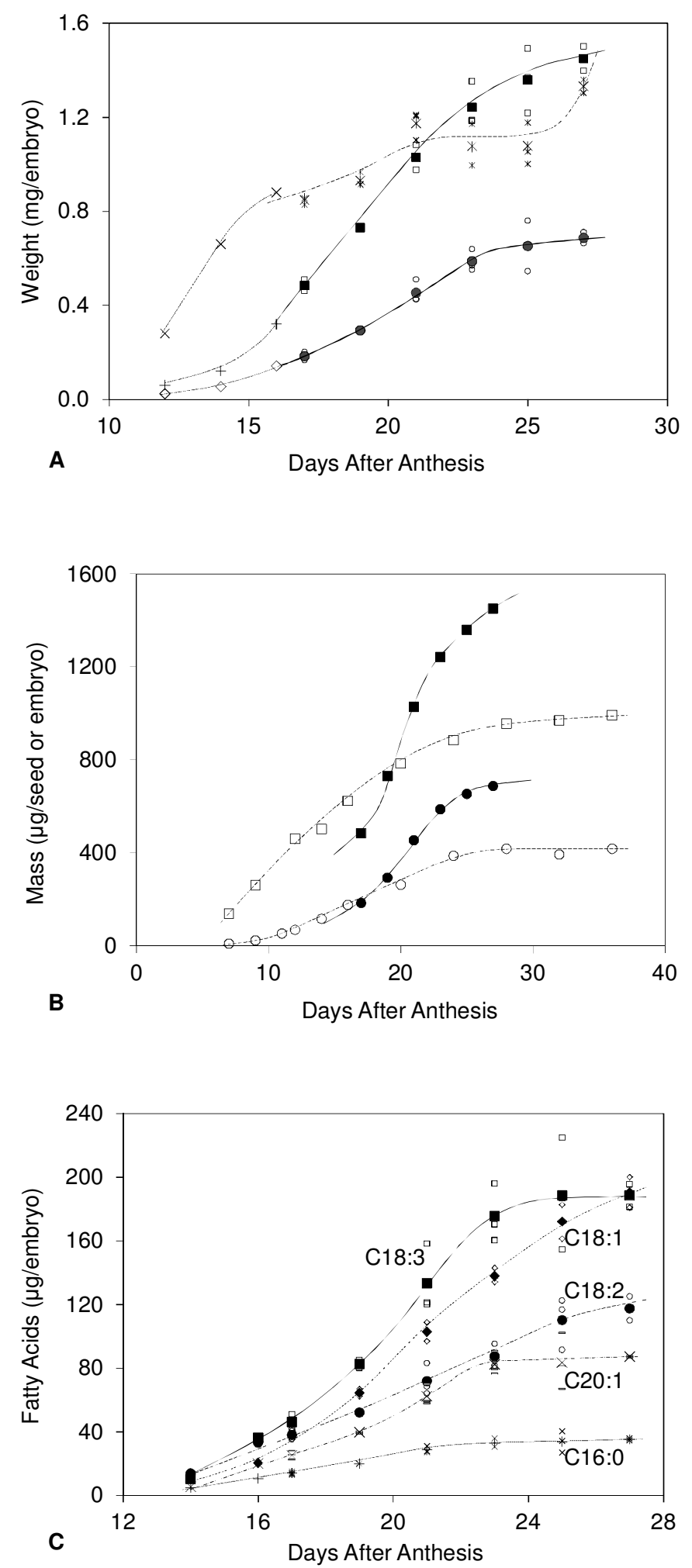
Figure 6
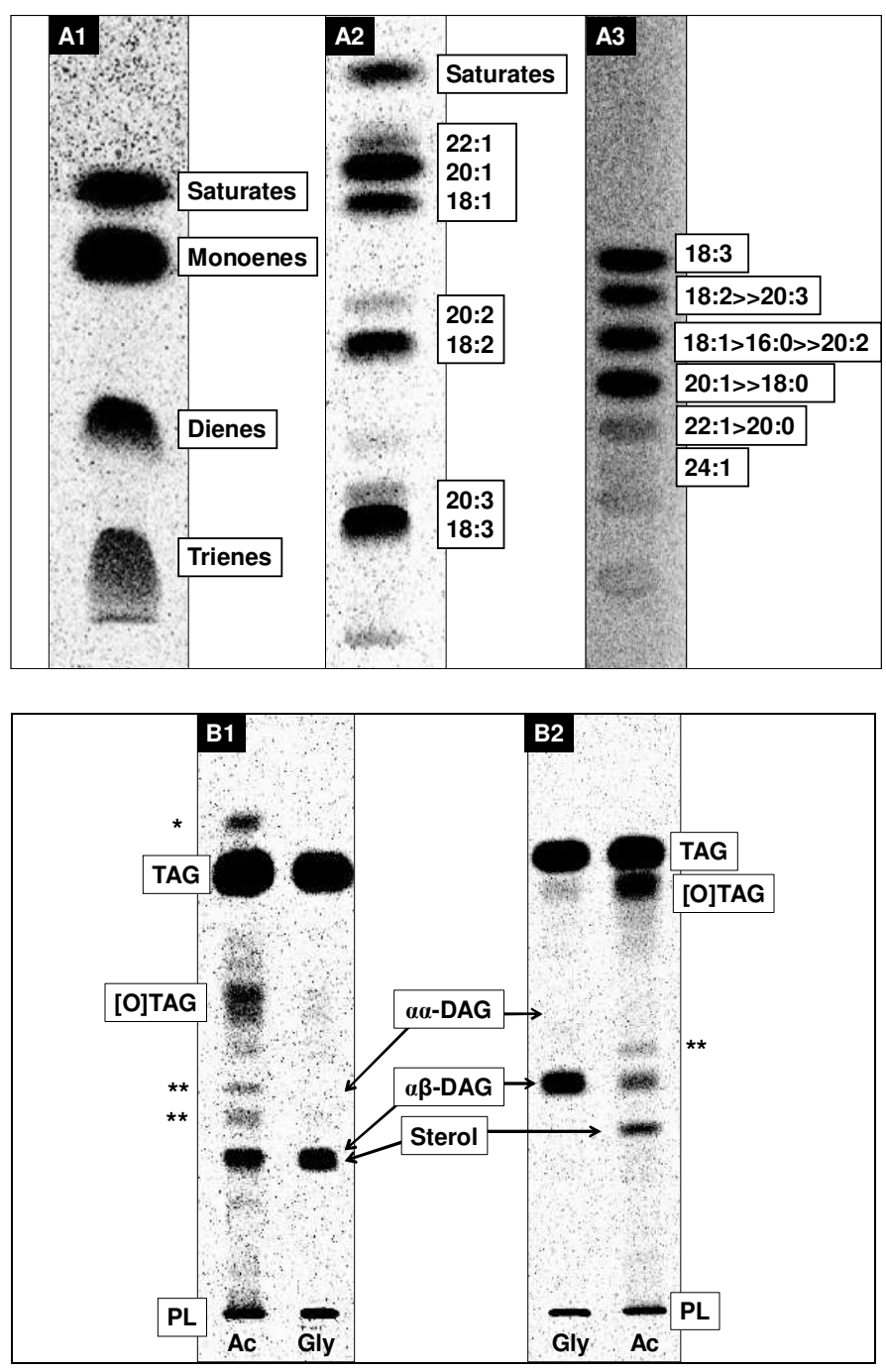


\section{Figure 7}

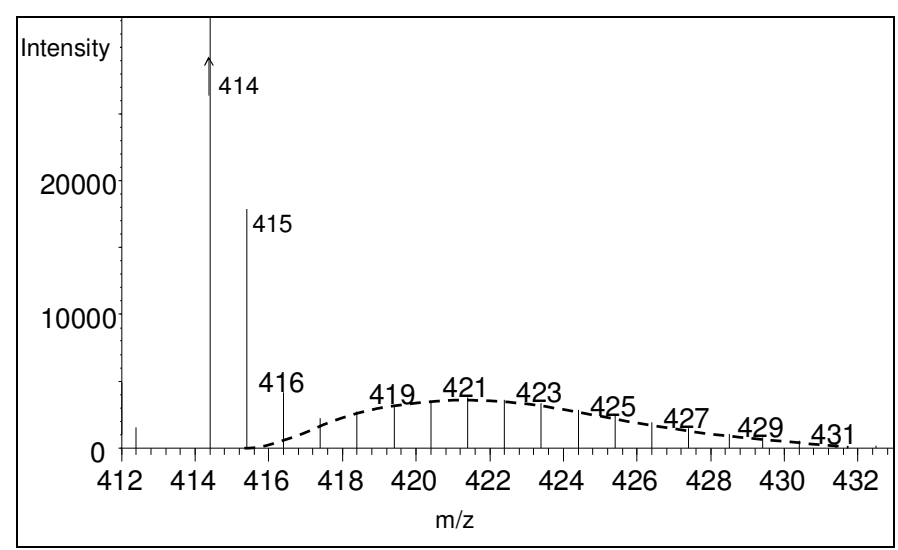




\section{Graphical Abstract}

\section{Lipid Analysis of Developing Camelina sativa Seeds and Cultured Embryos}

Mike Pollard, Tina M. Martin and Yair Shachar-Hill

Camelina sativa seed development in silique and the culture of developing embryos were compared with respect to lipid deposition. Fatty acid, triacylglycerol and lipid class analyses were optimized to resolve and identify minor components.

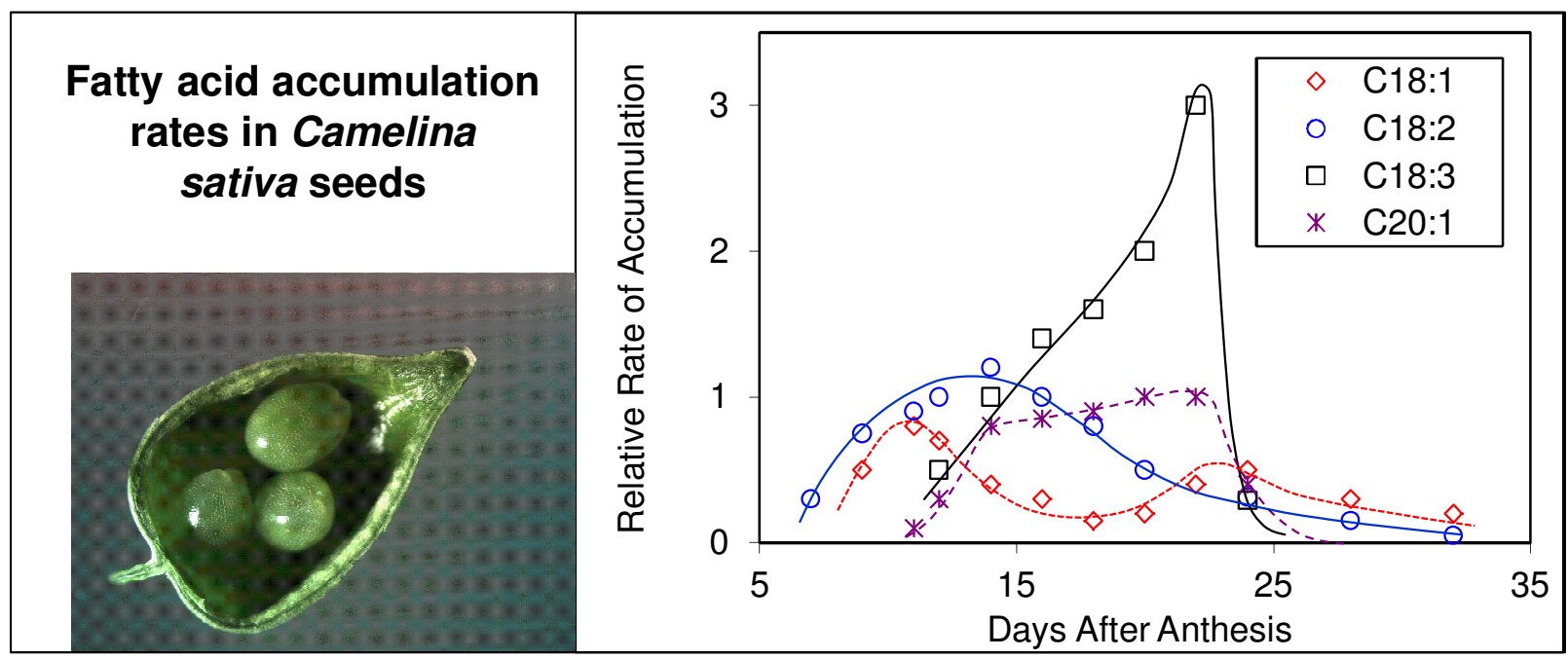

